\title{
Hashimoto's thyroiditis might increase polycystic ovary syndrome and associated comorbidities risks in Asia
}

\author{
Chun-Wei Ho ${ }^{1 \#}$, Hsin-Hung Chen ${ }^{1,2 \#}$, Ming-Chia Hsieh ${ }^{1,3,4}$, Ching-Chu Chen ${ }^{5,6}$, Sheng-Pang Hsu ${ }^{5}$, \\ Hei-Tung Yip ${ }^{7,8}$, Chia-Hung Kao, 10,11,12
}

${ }^{1}$ Intelligent Diabetes Metabolism and Exercise Center, China Medical University Hospital, Taichung; ${ }^{2}$ School of Medicine, Institute of Medicine and Public Health, Chung Shan, Medical University, Taichung; ${ }^{3}$ Graduate Institute of Integrative Medicine, China Medical University, Taichung; ${ }^{4}$ Division of Clinical Nutrition, ${ }^{5}$ Division of Endocrinology and Metabolism, Department of Internal Medicine, China Medical University Hospital, Taichung; ${ }^{6}$ School of Chinese Medicine, China Medical University, Taichung; ${ }^{7}$ Management Office for Health Data, China Medical University Hospital, Taichung; ${ }^{8}$ School of Medicine, College of Medicine, ${ }^{9}$ Graduate Institute of Biomedical Sciences, China Medical University, Taichung; ${ }^{10}$ Department of Nuclear Medicine and PET Center, China Medical University Hospital, Taichung; ${ }^{11}$ Department of Bioinformatics and Medical Engineering, Asia University, Taichung; ${ }^{12}$ Center of Augmented Intelligence in Healthcare, China Medical University Hospital, Taichung

Contributions: (I) Conception and design: HH Chen, CH Kao; (II) Administrative support: None; (III) Provision of study materials or patients: CH Kao; (IV) Collection and assembly of data: All authors; (V) Data analysis and interpretation: All authors; (VI) Manuscript writing: All authors; (VII) Final approval of manuscript: All authors.

\#These authors contributed equally to this work.

Correspondence to: Chia-Hung Kao, MD. Graduate Institute of Biomedical Sciences and School of Medicine, College of Medicine, China Medical University, No. 2, Yuh-Der Road, 404, Taichung. Email: d10040@mail.cmuh.org.tw; dr.kaochiahung@gmail.com.

Background: To investigate whether increased the comorbidities such as coronary artery disease (CAD) and risks between Hashimoto's thyroiditis (HT) and polycystic ovary syndrome (PCOS) in Taiwanese women.

Methods: Patients newly diagnosed as having HT during 2000-2012 were assigned to the case group. Cases and controls were matched for age and comorbidities at a 1:2 ratio using propensity score matching. Incidence was calculated in the unit of 1000 person-year. Univariate and multivariate Cox proportional hazard regression, multivariate Cox, logistic regression, and Kaplan-Meier analyses were performed.

Results: Among 3,996 participants, 2,664 constituted the control group and 1,332 constituted the case group. The PCOS risk in patients with HT increased by 2.37 times [95\% confidence interval (CI): 1.22-4.62] compared with the controls. Hypertension (HTN) [adjusted odds ratio (OR): 1.31, 95\% CI: 1.03-1.66] and hyperlipidemia (adjusted OR: 1.55, 95\% CI: 1.2-1.9) were more common in HT patients without PCOS than in other patients. The adjusted OR for CAD in patients with HT was 1.51 (95\% CI: 1.11-2.06), whereas that in patients with HT and PCOS was 5.92 (95\% CI: 1.32-26.53).

Conclusions: In our study, the PCOS risk in patients with HT increased by 2.37 times, which is lower than the increase in HT risk in Asian patients with PCOS (4.56 times). The proportion of CAD increased significantly by 5.92 times in patients with HT and PCOS compared with patients with HT only.

Keywords: Hashimoto's thyroiditis (HT); polycystic ovary syndrome (PCOS); coronary artery disease (CAD)

Submitted Dec 21, 2019. Accepted for publication May 09, 2020.

doi: 10.21037/atm-19-4763

View this article at: http://dx.doi.org/10.21037/atm-19-4763 


\section{Introduction}

Hashimoto's thyroiditis (HT) was first described by Hakaru Hashimoto in 1912, but the autoimmunity of HT was demonstrated in the 1950s. The etiology of HT is not completely known. According to epidemiological studies, HT is the most common autoimmune disease and also the most common endocrine disorder to cause hypothyroidism (1-3). HT was classified into primary and secondary forms according to its etiology (4). The clinicopathological spectrum of HT comprises six primary entities, such as the classic form and silent thyroiditis $(5,6)$. Lymphocytic infiltration of the thyroid gland is the predominant pathological feature. Secondary form is usually iatrogenic, which is commonly induced by immunomodulatory drugs, such as interferon-alpha for hepatitis $\mathrm{C}$ viral infection treatment and monoclonal antibodies blocking CTLA-4 for cancer therapy $(7,8)$. In general, HT is recognized as an organ-specific autoimmune disease with circulating antibodies, such as thyroperoxidase (TPO) and thyroglobulin (Tg), to thyroid antigens. This immune response can engender impaired conversion of estrogen precursors (4). High estrogen levels instead of androgens and progesterone are implicated in humoral immunity enhancement $(9,10)$. Polycystic ovary syndrome (PCOS) with an increased estrogen-to-progesterone ratio is the most common endocrine disorder of women, affecting $5-10 \%$ of all reproductive-aged women worldwide $(11,12)$. Studies have reported that among Whites and Asians, women are more likely to have HT than men $(4,13)$. Hence, we investigated the association between HT and PCOS in Taiwanese women, including the comorbidities such as diabetes, hypertension (HTN), coronary artery disease (CAD) and risks between both diseases. Previous studies investigating the association of PCOS with HT have always focused on PCOS; by contrast, we attempted to examine the association from the perspective of HT. We present the following article in accordance with the STROBE reporting checklist (available at http://dx.doi.org/10.21037/atm-194763).

\section{Methods}

\section{Data source}

In March 1995, the Taiwan National Health Insurance (NHI) program was initiated. The program provides health care coverage for approximately 99\% of Taiwan's population. The National Health Insurance Administration established the National Health Insurance Research Database (NHIRD), which is managed by the National Health Research Institute. The NHIRD includes health information such as health status, medication, or treatment of insured individuals. In this study, we used the Longitudinal Health Insurance Database, a sub-database of the NHIRD with 1 million randomly selected insured individuals. Diseases were coded according to International Classification of Diseases, Ninth Revision, Clinical Modification (ICD-9-CM) codes.

\section{Ethics statement}

The NHIRD encrypts patient personal information to protect privacy and provides researchers with anonymous identification numbers associated with relevant claims information, including sex, date of birth, medical services received, and prescriptions. Therefore, patient consent is not required to access the NHIRD. This study was approved to fulfill the condition for exemption by the Institutional Review Board (IRB) of China Medical University (CMUH104-REC2-115-CR4). The IRB also specifically waived the consent requirement.

\section{Study sample}

This cohort study included a case group and control group. Patients newly diagnosed as having HT (ICD-9-CM code: 245.21) during the 2000-2012 period were assigned to the case group. The definition of HT patients was subjects with two or more outpatient visits or one hospitalization record of HT diagnosis. Individuals without HT constituted the control group. We excluded individuals with a history of PCOS before entry in the study and those aged younger than 20 years from both groups. Cases and controls were matched according to age and index year, which was the date of HT diagnosis, at a 1:2 ratio using propensity score matching.

\section{Main event and covariates}

The endpoint of the present study was diagnosis of PCOS (ICD-9-CM code 2564). We considered the following comorbidities, which developed before the index date, as potential confounders of PCOS: diabetes mellitus (DM) (ICD-9-CM code 250), hyperlipidemia (ICD-9-CM code 272), HTN (ICD-9-CM codes 401-405), stroke (ICD-9CM codes 430-438), CAD (ICD-9-CM codes 410-414), 
Table 1 The baseline characters of patient with and without Hashimoto thyroiditis

\begin{tabular}{|c|c|c|c|c|c|}
\hline \multirow{3}{*}{ Variables } & \multicolumn{4}{|c|}{ Hashimoto thyroiditis } & \multirow{3}{*}{$P$ value } \\
\hline & \multicolumn{2}{|c|}{$\mathrm{No}=2,664$} & \multicolumn{2}{|c|}{ Yes=1,332 } & \\
\hline & $n$ & $\%$ & $\mathrm{n}$ & $\%$ & \\
\hline Age & & & & & 1.00 \\
\hline$<30$ & 510 & 19.0 & 255 & 19.0 & \\
\hline 30-39 & 632 & 24 & 316 & 24 & \\
\hline$\geq 40$ & 1,522 & 57 & 761 & 57 & \\
\hline Mean $(\mathrm{SD})^{\dagger}$ & 45.1 & 16.3 & 43.7 & 14.4 & $<0.001$ \\
\hline \multicolumn{6}{|l|}{ Comorbidities } \\
\hline DM & 275 & 10 & 175 & 13 & 0.01 \\
\hline HTN & 594 & 22 & 278 & 21 & 0.32 \\
\hline Hyperlipidemia & 433 & 16 & 298 & 22 & $<0.001$ \\
\hline Stroke & 162 & 6.1 & 94 & 7.1 & 0.26 \\
\hline CAD & 328 & 12 & 192 & 14 & 0.07 \\
\hline Heart failure & 67 & 2.5 & 37 & 2.8 & 0.70 \\
\hline PCOS & 16 & 0.6 & 19 & 1.4 & 0.01 \\
\hline
\end{tabular}

Chi-square test; ${ }^{\dagger}$ Student $t$-test. DM, diabetes mellitus; HTN, hypertension; CAD, coronary artery disease; PCOS, polycystic ovary syndrome.

and heart failure (ICD-9-CM codes 428, 428.0, 428.1, and 428.9).

\section{Statistical analysis}

A chi-square test was used to compare the baseline categorical variables between the case and control group. A Student $t$-test was used to assess mean of age between two groups. Incidence was calculated in the unit of 1,000 personyear. Univariate Cox proportional hazard models were used to estimate hazard ratios for PCOS. The multivariate Cox model included age and all comorbidities as variables to adjust the hazard ratio of PCOS. Logistic regression was then applied to investigate the effect of comorbidities on the occurrence of HT or PCOS. The cumulative incidence of PCOS in the case and control groups was evaluated using the Kaplan-Meier method. A Log-rank test was then used to verify the difference between the two curves. The statistical significance level was set as a $\mathrm{P}$ value less than 0.05 . All statistical analyses were performed by SAS (version 9.4;
SAS Institute, Inc., Cary, NC, USA).

\section{Results}

Of 3,996 participants included in this study, 2,664 constituted the control group and 1,332 constituted the case group. The follow-up time for the case group was 6.69 $( \pm 3.81)$ years, and that for the control group was $6.69( \pm 3.77)$ years. Most of the participants were aged older than 40 years. The mean ages in the control group was $45.1( \pm 16.3)$ years, and that in the case group was $43.7( \pm 14.4)$ years. The case group had higher proportions of HTN and hyperlipidemia. In the case and control groups $1.4 \%(\mathrm{n}=19)$ and $0.6 \%(\mathrm{n}=16)$ of the participants developed PCOS (Table 1).

Table 2 lists the incidence and hazard ratios of PCOS. The PCOS risk in patients with HT increased by 2.37 times [95\% confidence interval (CI): 1.22-4.62] compared with that in participants without HT. The risk of PCOS decreased by 0.88 -fold (95\% CI: $0.85-0.92$ ) with an increase in age. Comorbidities were not statistically significant risk factors for PCOS.

The results of comorbidity-specific analysis are listed in Table 3. HTN [adjusted odds ratio (OR): 1.31, 95\% CI: 1.03-1.66] and hyperlipidemia (adjusted OR: 1.55, 95\% CI: 1.2-1.9) were more common in HT patients without PCOS than in the controls. The adjusted OR for CAD in patients with HT was 1.51 (95\% CI: 1.11-2.06), and that in patients with HT and PCOS was 5.92 (95\% CI: 1.32-26.53).

The cumulative incidence of PCOS in patients with HT was higher than that in the controls, and the difference was statistically significant $(\mathrm{P}=0.009$; Figure 1$)$.

\section{Discussion}

\section{Associated risks between HT and PCOS}

In our study, the risk of PCOS in patients with HT increased by 2.37 times (95\% CI: 1.22-4.62) compared with participants without HT. Compared with other studies on HT risk in PCOS, the general risk was higher by 4.56 times in Asians, 3.27 times in Europeans, and 1.86 times in South Americans (14). One prospective study demonstrated a 3 times higher prevalence of autoimmune thyroiditis in the PCOS group compared with the control group (15). Another recent study on Danish women revealed that the event rate of thyroid disease increased significantly by 2.5 times in the PCOS group (16). Compared with other studies, although our analysis was based on clinical 
Table 2 The incidence rate and hazard ratio of polycystic ovary syndrome

\begin{tabular}{|c|c|c|c|c|c|c|c|}
\hline Variables & \multicolumn{3}{|c|}{ PCOS } & \multicolumn{2}{|c|}{ Crude } & \multicolumn{2}{|c|}{ Adjusted } \\
\hline \multicolumn{8}{|l|}{ HT } \\
\hline No & 16 & 17,831 & 0.90 & 1.00 & Reference & 1.00 & Reference \\
\hline Yes & 19 & 8,917 & 2.13 & 2.37 & $1.22-4.62^{*}$ & 2.37 & $1.21-4.64^{\star}$ \\
\hline$<30$ & 25 & 5,489 & 4.55 & 1.00 & Reference & & \\
\hline 30-39 & 9 & 6,805 & 1.32 & 0.29 & $0.14-0.63^{\star \star \star}$ & & \\
\hline$\geq 40$ & 1 & 14,454 & 0.07 & 0.01 & $0.00-0.11^{* * *}$ & & \\
\hline \multicolumn{8}{|c|}{ Comorbidities } \\
\hline
\end{tabular}

Adjusted HR: adjusted with age and comorbidities of DM, HTN, hyperlipidemia, stroke, CAD and heart failure. Rate ${ }^{\#}$, incidence rate per 10,000 person-years. ${ }^{*}, \mathrm{P}<0.05$; ${ }^{\star \star \star}, \mathrm{P}<0.001$. Cl, confidence interval; HR, hazard ratio; PY, person-years; DM, diabetes mellitus; HTN, hypertension; CAD, coronary artery disease; PCOS, polycystic ovary syndrome; HT, Hashimoto's thyroiditis.

diagnoses using only ICD-9-CM codes and without any laboratory information, the accuracy of HT and PCOS diagnoses was acceptable. One of the reasons is that in the Taiwan NHI system, most patients, including those with HT and PCOS, are offered quick treatment by endocrinology and gynecology specialists without being transferred from primary physicians. Younger women aged younger than 30 years were the predominant group in our analysis, indicating that our government should pay more attention to these reproductive-aged women to improve aging in Taiwan.

\section{Associated comorbidities between HT and PCOS}

In general, overall comorbidities were not statistically significant. In our subgroup analysis, patients with HT only appeared to have more comorbidities, namely HTN, hyperlipidemia, and CAD, than did those with PCOS only. HT caused hypothyroidism or subclinical hypothyroidism and it resulted in hyperlipidemia (2.3) Notably, CAD was 5.92 times significantly higher in the HT patients with PCOS compared with other patients, strongly suggesting for early CAD screening in patients with HT and PCOS. The PCOS standard treatment guidelines indicate the importance of early diagnosis along with early intervention to improve not only infertility but also other risks of diabetes, HTN, and dyslipidemia for cardiovascular disease (CVD) $(17,18)$. To our knowledge, PCOS was associated with metabolic syndrome, insulin resistance, diabetes, obesity, dyslipidemia, HTN, and CVD $(14,19,20)$, and the common mechanisms were higher insulin level or low grade chronic inflammation due to high estrogen secretion influenced by the hypothalamicpituitary-gonadal axis (21). The association between inflammation and autoimmunity in PCOS was extensively discussed in a recent study (22). Whether stimulating ovarian antibodies in granulosa cells play a major role in PCOS pathophysiology (18) or the concept of nonorganspecific autoantibodies in PCOS (20) constitute the autoimmune etiology is still controversial $(23,24)$. These discussions partially explain the observation of the recurrent association between PCOS and autoimmune diseases such as Cushing syndrome and autoimmune thyroid diseases, the most common autoimmune disorder in the world $(25,26)$. HT, involving positive antibodies for TPO or $\mathrm{Tg}$, is one of the most common causes of hypothyroidism in young women through the mechanism of $\mathrm{T}$ cell-mediated organ-specific autoimmnity (27-29). One study revealed the relationship between subclinical hypothyroidism and PCOS with metabolic abnormalities, such as the level of high-density lipoprotein and homeostatic model assessment for insulin resistance (30). Regardless of study design, such 
Table 3 The odd ratios of comorbidities in patients with and without Hashimoto thyroiditis and polycystic ovary syndrome

\begin{tabular}{|c|c|c|c|}
\hline \multirow{2}{*}{ Variables } & \multirow{2}{*}{ Event, N (HT/PCOS) } & \multicolumn{2}{|c|}{ Adjusted } \\
\hline & & OR & $95 \% \mathrm{Cl}$ \\
\hline \multicolumn{4}{|l|}{ DM } \\
\hline No/no & $2,648 / 187$ & 1.00 & Reference \\
\hline Yes/no & $1,313 / 83$ & 0.95 & $0.73-1.25$ \\
\hline No/yes & $16 / 1$ & 1.81 & $0.23-13.92$ \\
\hline Yes/yes & $19 / 2$ & 3.03 & $0.69-13.41$ \\
\hline \multicolumn{4}{|l|}{ HTN } \\
\hline No/no & $2,648 / 196$ & 1.00 & Reference \\
\hline Yes/no & $1,313 / 120$ & 1.31 & $1.03-1.66^{*}$ \\
\hline No/yes & $16 / 2$ & 2.76 & $0.62-12.38$ \\
\hline Yes/yes & $19 / 1$ & 1.04 & $0.14-7.85$ \\
\hline \multicolumn{4}{|c|}{ Hyperlipidemia } \\
\hline No/no & $2,648 / 263$ & 1.00 & Reference \\
\hline Yes/no & $1,313 / 185$ & 1.55 & $1.27-1.9^{\star \star \star}$ \\
\hline No/yes & $16 / 0$ & - & - \\
\hline Yes/yes & $19 / 1$ & 0.74 & $0.1-5.62$ \\
\hline \multicolumn{4}{|l|}{ Stroke } \\
\hline No/no & $2,648 / 103$ & 1.00 & Reference \\
\hline Yes/no & $1,313 / 57$ & 1.32 & $0.94-1.85$ \\
\hline No/yes & $16 / 0$ & - & - \\
\hline Yes/yes & $19 / 1$ & 4.86 & $0.63-37.73$ \\
\hline \multicolumn{4}{|l|}{ CAD } \\
\hline No/no & $2,648 / 108$ & 1.00 & Reference \\
\hline Yes/no & $1,313 / 73$ & 1.51 & $1.11-2.06^{\star *}$ \\
\hline No/yes & $16 / 0$ & - & - \\
\hline Yes/yes & $19 / 2$ & 5.92 & $1.32-26.53^{*}$ \\
\hline \multicolumn{4}{|c|}{ Heart failure } \\
\hline No/no & $2,648 / 77$ & 1.00 & Reference \\
\hline Yes/no & $1,313 / 28$ & 0.91 & $0.58-1.43$ \\
\hline No/yes & $16 / 0$ & - & - \\
\hline Yes/yes & $19 / 0$ & - & - \\
\hline
\end{tabular}

Adjusted OR: adjusted with age. ${ }^{*}, \mathrm{P}<0.05 ;{ }^{* *}, \mathrm{P}<0.01$; ${ }^{* *}$, $\mathrm{P}<0.001$. DM, diabetes mellitus; HTN, hypertension; CAD, coronary artery disease; PCOS, polycystic ovary syndrome; HT, Hashimoto's thyroiditis; OR, odds ratio.

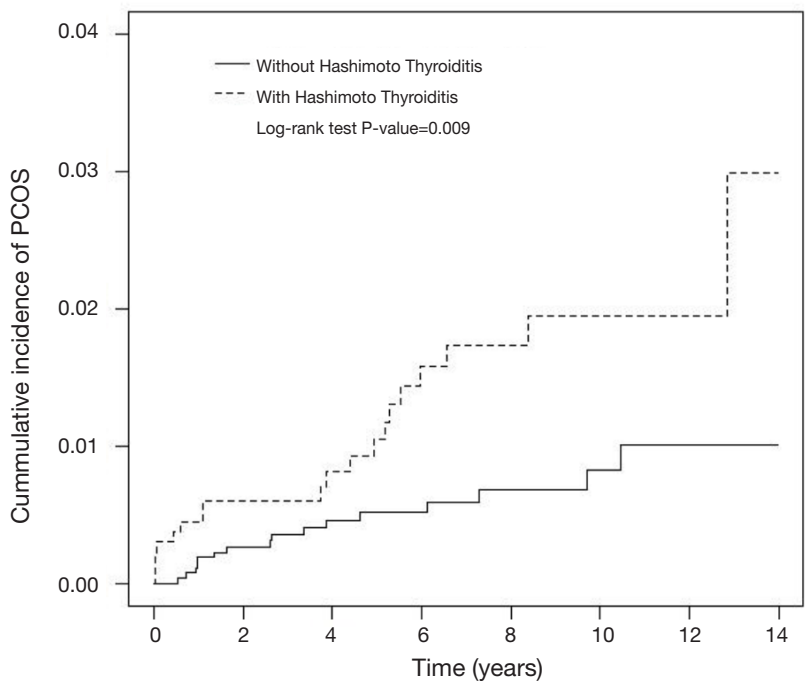

Figure 1 The cumulative incidence of PCOS (polycystic ovary syndrome) in patients with HT was higher than that in the controls, and the difference was statistically significant $(\mathrm{P}=0.009)$. PCOS, polycystic ovary syndrome; HT, Hashimoto's thyroiditis.

as autoimmunity or epidemiology designs, the association between HT and PCOS still remains unclear.

\section{Limitations}

First, our national database lacks laboratory information such as TSH, FT4, FT3, anti-TPO, anti-TG levels for $\mathrm{HT}$, insulin and fasting glucose level for insulin resistance calculation, lipid profile with cholesterol or triglyceride and estrogen, progesterone, and androgen levels for PCOS; therefore, we could not include such information in our analysis. In addition, we did not conduct an imaging study, such as thyroid or ovary echograms. Second, lifestyle or other environmental factors such as nutritional state, other pollution, smoking, alcohol consumption, and exercise habits were not included in the study.

\section{Conclusions}

In our study, the risk of PCOS in patients with HT increased by 2.37 times, which is lower than the increase in the risk of HT in Asian patients with PCOS (4.56 times). The proportion of CAD increased significantly by 5.92 times in patients with HT and PCOS compared with 
patients with HT only. Considering the public health issue, infertility as well as CAD screening should be indicated for patients with both HT and PCOS.

\section{Acknowledgments}

Funding: This study is supported in part by Taiwan Ministry of Health and Welfare Clinical Trial and Research Center of Excellence (MOHW108-TDU-B-212-133004); China Medical University Hospital (DMR-107-192, CMU107ASIA-19); Academia Sinica Stroke Biosignature Project (BM10701010021); MOST Clinical Trial Consortium for Stroke (MOST 108-2321-B-039-003-); Tseng-Lien Lin Foundation, Taichung; and Katsuzo and Kiyo Aoshima Memorial Funds, Japan. The funders had no role in the study design, data collection and analysis, the decision to publish, or preparation of the manuscript. No additional external funding was received for this study.

\section{Footnote}

Reporting Checklist: The authors have completed the STROBE reporting checklist. Available at http://dx.doi. org/10.21037/atm-19-4763

Data Sharing Statement: Available at http://dx.doi. org/10.21037/atm-19-4763

Conflicts of Interest: All authors have completed the ICMJE uniform disclosure form (available at http://dx.doi. org/10.21037/atm-19-4763). The authors have no conflicts of interest to declare.

Ethical Statement: The authors are accountable for all aspects of the work in ensuring that questions related to the accuracy or integrity of any part of the work are appropriately investigated and resolved. This study was approved to fulfill the condition for exemption by the Institutional Review Board (IRB) of China Medical University (CMUH104-REC2-115-CR4). The IRB also specifically waived the consent requirement.

Open Access Statement: This is an Open Access article distributed in accordance with the Creative Commons Attribution-NonCommercial-NoDerivs 4.0 International License (CC BY-NC-ND 4.0), which permits the noncommercial replication and distribution of the article with the strict proviso that no changes or edits are made and the original work is properly cited (including links to both the formal publication through the relevant DOI and the license). See: https://creativecommons.org/licenses/by-nc-nd/4.0/.

\section{References}

1. McLeod DS, Cooper DS. The incidence and prevalence of thyroid autoimmunity. Endocrine 2012;42:252-65

2. Golden SH, Robinson KA, Saldanha I, et al. Clinical review: prevalence and incidence of endocrine and metabolic disorders in the United States: a comprehensive review. J Clin Endocrinol Metab 2009;94:1853-78.

3. Delemer B, Aubert JP, Nys P, et al. An observational study of the initial anagement of hypothyroidism in France: the ORCHIDEE study. Eur J Endocrinol 2012;167:817-23.

4. Caturegli P, De Remigis A, Rose NR. Hashimoto thyroiditis: clinical and diagnostic criteria. Autoimmun Rev 2014;13:391-7.

5. Caturegli P, De Remigis A, Chuang K, et al. Hashimoto's thyroiditis: celebrating the centennial through the lens of the Johns Hopkins hospital surgical pathology records. Thyroid 2013;23:142-50.

6. Stagnaro-Green A. Approach to the patient with postpartum thyroiditis. J Clin Endocrinol Metab 2012;97:334-42.

7. Mandac JC, Chaudhry S, Sherman KE, et al. The clinical and physiological spectrum of interferon-alpha induced thyroiditis: toward a new classification. Hepatology 2006;43:661-72.

8. Corsello SM, Barnabei A, Marchetti P, et al. Endocrine side effects induced by immune checkpoint inhibitors. J Clin Endocrinol Metab 2013;98:1361-75.

9. Paavonen T. Hormonal regulation of immune responses. Ann Med 1994;26:255-8.

10. Ahmed SA, Hissong BD, Verthelyi D, et al. Gender and risk of autoimmune diseases: possible role of estrogenic compounds. Environ Health Perspect 1999;107:681-6.

11. Knochenhauer ES, Key TJ, Kahsar-Miller M, et al. Prevalence of the polycystic ovary syndrome in unselected black and white women of the southeastern United States: a prospective study. J Clin Endocrinol Metab 1998;83:3078-82.

12. Asunción M, Calvo RM, San Millan JL, et al. A prospective study of the prevalence of the polycystic ovary syndrome in unselected Caucasian women from Spain. J Clin Endocrinol Metab 2000;85:2434-8.

13. Hollowell JG, Staehling NW, Flanders WD, et al. Serum TSH, T(4), and thyroid antibodies in the United States 
population (1988 to 1994): National Health and Nutrition Examination Survey (NHANES III). J Clin Endocrinol Metab 2002;87:489-99.

14. Romitti M, Fabris VC, Ziegelmann PK, et al. Association between PCOS and autoimmune thyroid disease: a systematic review and meta-analysis. Endocr Connect 2018;7:1158-67.

15. Janssen OE, Mehlmauer N, Hahn S, et al. High prevalence of autoimmune thyroiditis in patients with polycystic ovary syndrome. Eur J Endocrinol 2004;150:363-9.

16. Glintborg D, Rubin KH, Nybo M, et al. Increased risk of thyroid disease in Danish women with polycystic ovary syndrome: a cohort study. Endocr Connect 2019;8:1405-15.

17. Goodman NF, Cobin RH, Futterweit W, et al. American association of clinical endocrinologists, American college of endocrinology, and androgen excess and PCOS society disease state clinical review: guide to the best practices in the evaluation and treatment of polycystic ovary syndrome. Endocr Pract 2015;21:1291-300.

18. Legro RS, Arslanian SA, Ehrmann DA, et al. Diagnosis and treatment of polycystic ovary syndrome: an Endocrine Society clinical practice guideline J Clin Endocrinol Metab 2013;98:4565-92.

19. Goverde AJ, van Koert AJ, Eijkemans MJ, et al. Indicators for metabolic disturbances in anovulatory women with polycystic ovary syndrome diagnosed according to the Rotterdam consensus criteria. Hum Reprod 2009;24:710-7.

20. Wild RA, Carmina E, Diamanti-Kandarakis E, et al. Assessment of cardiovascular risk and preventionof cardiovascular disease in women with the polycystic ovary syndrome: a consensus statement by the Androgen Excess andPolycystic Ovary Syndrome (AE-PCOS) Society. J Clin Endocrinol Metab 2010;95:2038-49.

Cite this article as: Ho CW, Chen HH, Hsieh MC, Chen CC, Hsu SP, Yip HT, Kao CH. Hashimoto's thyroiditis might increase polycystic ovary syndrome and associated comorbidities risks in Asia. Ann Transl Med 2020;8(11):684. doi: 10.21037/ atm-19-4763
21. Spritzer PM, Lecke SB, Satler F, et al. Adipose tissue dysfunction, adipokines, and low-grade chronic inflammation in polycystic ovary syndrome. Reproduction 2015;149:R219-27.

22. Sen A, Kushnir VA, Barad DH, et al. Endocrine autoimmune diseases and female infertility. Nat Rev Endocrinol 2014;10:37-50.

23. van Gelderen CJ, Gomes dos Santos ML. Polycystic ovarian syndrome. Evidence for an autoimmune mechanism in some cases. J Reprod Med 1993;38:381-6.

24. Hefler-Frischmuth K, Walch K, Huebl W, et al. Serologic markers of autoimmunity in women with polycystic ovary syndrome. Fertil Steril 2010;93:2291-4.

25. Kaltsas GA, Korbonits M, Isidori AM, et al. How common are polycystic ovaries and the polycystic ovarian syndrome in women with Cushing's syndrome? Clin Endocrinol (Oxf) 2000;53:493-500.

26. Jacobson DL, Gange SJ, Rose NR, et al. Epidemiology and estimated population burden of selected autoimmune diseases in the United States. Clin Immunol Immunopathol 1997;84:223-43.

27. Antonelli A, Ferrari SM, Corrado A, et al. Autoimmune thyroid disorders. Autoimmun Rev 2015;14:174-80.

28. Dayan CM, Daniels GH. Chronic autoimmune thyroiditis. N Engl J Med 1996;335:99-107.

29. Cooper DS. Clinical practice. Subclinical hypothyroidism. N Engl J Med 2001;345:260-5.

30. Pergialiotis V, Konstantopoulos P, Prodromidou A, et al. MANAGEMENT OF ENDOCRINE DISEASE: the impact of subclinical hypothyroidism on anthropometric characteristics, lipid, glucose and hormonal profile of PCOS patients: a systematic review and meta-analysis. Eur J Endocrinol 2017;176:R159-66. 